\title{
Possibilities for a specific breeding program for organic dairy production
}

\author{
M. Slagboom, ${ }^{1 *} \odot$ L. Hjortø, ${ }^{1}$ A. C. Sørensen, ${ }^{1}$ H. A. Mulder, ${ }^{2} \odot$ J. R. Thomasen,,${ }^{1,3}$ and M. Kargo ${ }^{1,4}{ }^{\circ}$ \\ ${ }^{1}$ Department of Molecular Biology and Genetics, Center for Quantitative Genetics and Genomics, Aarhus University, 8830 Tjele, Denmark \\ ${ }^{2}$ Wageningen University and Research Animal Breeding and Genomics, $6700 \mathrm{AH}$ Wageningen, the Netherlands \\ ${ }^{3}$ VikingGenetics, 8960 Assentoft, Denmark \\ ${ }^{4}$ SEGES Cattle, 8200 Aarhus N, Denmark
}

\begin{abstract}
Organic dairy production differs from conventional dairy production in many aspects. However, breeding programs for the 2 production systems are the same in most countries. Breeding goals (BG) might be different for the 2 production systems and genotype $x$ environment interaction may exist between organic and conventional dairy production, both of which have an effect on genetic gain in different breeding strategies. Other aspects also need to be considered, such as the application of multiple ovulation and embryo transfer (MOET), which is not allowed in organic dairy production. The general aim of this research was to assess different environment-specific breeding strategies for organic dairy production. The specific aim was to study differences in BG weights and include the effect of genotype $\times$ environment interaction, MOET, and the selection of breeding bulls from the conventional environment. Different scenarios were simulated. In the current scenario, the present-day situation for dairy production in Denmark was emulated as much as possible. The BG was based on a conventional dairy production system, MOET was applied in both environments, and conventional bulls could be selected as breeding bulls in the organic environment. Four alternative scenarios were simulated, all with a specific organic BG in the organic breeding program but differences in the usage of MOET and the selection of conventional bulls as breeding bulls. Implementation of a specific BG in organic dairy production slightly increased genetic gain in the aggregate genotype compared with the breeding program that is currently implemented in organic dairy production. Not using embryo transfer or only selecting breeding bulls from the organic environment decreased genetic gain in the aggregate genotype by as much as
\end{abstract}

Received May 3, 2019.

Accepted February 12, 2020.

*Corresponding author: margotslagboom@gmail.com
$24 \%$. However, the use of embryo transfer is debatable because this is not allowed according to current regulations for organic dairy production. Assessing genetic gain on trait levels showed that a significant increase for functional traits was possible compared with the current breeding program in the organic environment without a decrease in genetic gain in the aggregate genotype. This difference on trait level was even more present when selection of conventional bulls as breeding bulls in the organic environment was not possible. This finding is very relevant when breeding for the desired cow in organic dairy production.

Key words: genetic gain, dairy cow, breeding strategy, organic production

\section{INTRODUCTION}

Organic dairy production differs from conventional dairy production in many aspects. However, breeding programs for the 2 production systems are the same in most countries. In some countries, examples of research and implementation of organic selection indices can be found (Krogmeier, 2003; Bapst et al., 2005; Rozzi et al., 2007). However, in Denmark and the other Nordic countries, the current economic values used in the Nordic Total Merit index (NTM) are based on an average production environment (Kargo et al., 2014). Approximately $12 \%$ of dairy cows in Denmark are organic (SEGES, 2018). Therefore, economic values based on an average production environment may not sufficiently consider the organic environment. Slagboom et al. (2018) simulated long-term effects of breeding goals (BG) specifically for organic dairy production based on different methods. Breeding goals based on economic models for organic dairy production (Kargo et al., 2015) or based on preferences of organic farmers (Slagboom et al., 2016) were very similar and had very high $\mathrm{BG}$ weights on production traits. These $\mathrm{BG}$ caused favorable genetic change in production traits but also unfavorable genetic change in functional traits. However, the newest NTM calculations (Sørensen et al., 2018) include economic values calculated specifi- 
cally for organic production, and these economic values have higher weights on functional traits. Alternatively, BG designed to resemble the principles of organic agriculture (IFOAM, 2016) or BG set up with desired gain indices aimed at improving functional traits seemed more suitable for organic dairy production (Slagboom et al., 2018). However, these BG might be lacking in economic progress, and farmer acceptance of such BG is questionable. In addition, looking solely at differences in BG weights is not sufficient to justify setting up different breeding programs for the 2 production systems.

Genotype $\times$ environment interaction $(\mathbf{G} \times \mathbf{E})$ may exist between organic and conventional dairy production. Previous studies have shown that moderate $\mathrm{G} \times \mathrm{E}$ between organic and conventional dairy production exists (Nauta et al., 2006; Sundberg et al., 2010). Both $\mathrm{G} \times \mathrm{E}$ and differences in BG weights lower the genetic correlation between BG for the 2 environments, which is an important indicator of the necessity for separate breeding programs for different environments. The so-called break-even correlation indicates the specific correlation point above which it is better with regard to genetic gain to apply 1 breeding program for 2 environments (Mulder et al., 2006). Mulder (2007) suggested that the break-even correlation will be higher with genomic selection due to a possible higher intensity of selection. Slagboom et al. (2019) showed by stochastic simulation that the break-even correlation is higher with genomic selection if the selection intensity is higher, but with the same selection intensity, the break-even correlation is the same. Differences in BG weights and $\mathrm{G} \times \mathrm{E}$ need to be combined to estimate the correlation between $\mathrm{BG}$ for organic and conventional dairy production.

Other aspects of the breeding program also need to be considered, such as decisions relating to the use of reproductive technologies such as multiple ovulation and embryo transfer (MOET; Nicholas and Smith, 1983). Implementation of genomic breeding programs with MOET can increase genetic gain (Thomasen et al., 2016). However, MOET is not allowed in organic dairy production (Council of the European Union, 2018; IFOAM, 2018). In Denmark, using semen from bulls that are the product of MOET is allowed in organic dairy production, and this is commonly used in practice. If regulations become stricter, resulting in the exclusive use of bulls that are not the product of MOET, it might be necessary to use only bulls from organic environments. This can influence genetic gain in the aggregate genotype and on trait levels. The general aim of this research was to assess different environment-specific breeding strategies for organic dairy production for Holstein and Jersey cattle. These 2 breeds were included due to differences in population size and size of the reference population of both breeds (SEGES, 2018). The specific aim was to study differences in $B G$ weights and to include the effect of $\mathrm{G} \times \mathrm{E}$, MOET, and the selection of breeding bulls from the conventional environment.

\section{MATERIALS AND METHODS}

\section{Scenarios}

In this study, differences in $\mathrm{BG}$ weights, $\mathrm{G} \times \mathrm{E}$, and breeding strategies were combined in different scenarios to resemble a large breeding program (based on Nordic Holstein) and a small breeding program (based on Nordic Jersey). In all scenarios, 1 organic and 1 conventional environment were simulated. Three traits were included in the BG for each environment: cow fertility, mastitis, and milk production. In scenario Current, 1 breeding strategy was simulated for both environments that emulated the current situation for dairy production in Denmark. The BG was based on a conventional dairy production system. In this scenario, MOET was applied in both environments and conventional bulls could be selected as breeding bulls in the organic environment. Four alternative scenarios were simulated, all with a different breeding strategy in the organic environment compared with in the conventional environment. The same breeding strategy was applied to the conventional environment in all alternative scenarios, but different scenarios with different breeding strategies were applied in the organic environment (Table 1). All alternative scenarios had a specific organic BG in the organic breeding program but differed in the application of MOET and the selection of conventional bulls as breeding bulls in the organic breeding program. In scenario Organic BG, both MOET and the selection of conventional bulls were applied. In scenario Within, no conventional bulls could be selected in the organic breeding program. In scenario No MOET, no MOET was applied in the organic environment, and in scenario Strict, neither selection of conventional bulls nor MOET were applied.

Input variables for each breeding program differed per breed and for the organic and conventional environments. The breeding programs used in the simulated scenarios are shown in Figure 1 for Holstein and in Figure 2 for Jersey. Input parameters were based on the breeding programs used in practice for the Nordic Holstein and Nordic Jersey breeds. A breeding nucleus was simulated in each environment consisting of cows with ages 1 to 5 yr. Each year, several animals were genotyped in each environment based on parent average. Breeding bulls were selected from genotyped bulls 1 yr 
Table 1. Overview of scenarios applied to the organic breeding program $^{1}$

\begin{tabular}{llll}
\hline Scenario $^{2}$ & Breeding goal & MOET & $\begin{array}{l}\text { Selection of } \\
\text { conventional bulls }\end{array}$ \\
\hline Current & Conventional & Yes & Yes \\
Organic BG & Organic & Yes & Yes \\
Within & Organic & Yes & No \\
No MOET & Organic & No & Yes \\
Strict & Organic & No & No \\
\hline
\end{tabular}

${ }^{1}$ The scenario applied to the conventional breeding program was always equal to the scenario Current.

${ }^{2}$ Current $=$ a conventional breeding goal (BG), multiple ovulation and embryo transfer (MOET), and the possibility to select conventional bulls in the organic breeding program; Organic BG = an organic BG, MOET, and the possibility to select conventional bulls in the organic breeding program; Within = an organic BG, MOET, and no possibility to select conventional bulls in the organic breeding program; No $\mathrm{MOET}=$ an organic BG, no MOET, and the possibility to select conventional bulls in the organic breeding program; Strict $=$ an organic $\mathrm{BG}$, no MOET, and no possibility to select conventional bulls in the organic breeding program.

of age. In scenario Current, all selection decisions were performed in 1 selection step. In the other scenarios, 2 selection steps were applied for all selection decisions, 1 for each environment, to be able to select based on different BG. In this case, the total number of animals to be genotyped and the number of selected donors for MOET were equally divided between environments. In scenarios Within and Strict, bulls to be mated to cows in the organic environment could be selected only from bulls born in the organic environment. Heifers were selected for MOET for each environment from genotyped heifers of $1 \mathrm{yr}$ of age, except for in scenarios No MOET and Strict, where MOET was not allowed.

Scenarios were simulated for $30 \mathrm{yr}$ in the stochastic simulation program ADAM (Pedersen et al., 2009) and replicated 50 times. A setup using pseudogenomic selection was used (Buch et al., 2009). This means that no markers or QTL were simulated, but instead a correlated trait was simulated for each trait included in the simulation (based on the method by Dekkers, 2007). The correlation between this genomic trait and the biological trait was equal to the accuracy of DGV for that specific trait. In addition, each trait was simulated as a separate trait in the organic environment and in the conventional environment, meaning that for every biological trait, 4 traits were simulated.

\section{Genetic Parameters}

Trait parameters for the 3 traits included in the simulation were based on the female fertility index, udder health index, and standardized milk production used in the NTM. In the NTM, more than half of the
BG weight is on functional traits, $30 \%$ on production traits, and the rest on conformation (VikingGenetics, 2020). The female fertility index includes interval from calving to first insemination and interval from first to last insemination, the udder health index includes frequencies of treatments for different lactation periods, and standardized milk production is milk production standardized for $4.2 \%$ fat and $3.4 \%$ protein (Sørensen et al., 2018). All genetic and residual correlations between traits were assumed to be the same for the Holstein and Jersey breeds (Table 2). Residual correlations were calculated based on genetic and phenotypic correlations and variances. Correlations between traits in the same environment were based on estimates from the literature and rounded to the nearest 0.05 points (Kadarmideen et al., 2000; Buch and Norberg, 2008). These correlations were assumed to be the same for the organic and the conventional environment. Residual correlations between traits in different environments were zero. Genetic correlations between the same trait in the organic and the conventional environment were based on the $\mathrm{G} \times \mathrm{E}$ estimation by A. Liu (Aarhus University, Tjele, Denmark, personal communication; Liu et al., 2019). Genetic correlations between one trait in one environment and another trait in the other environment were calculated using Equation 1:

$$
r_{i 1, j 2}=\frac{r_{i 1, i 2} \times r_{i, j}+r_{i, j} \times r_{j 1, j 2}}{2}
$$

where $r_{i 1, j 2}=$ the genetic correlation between trait $i$ in environment 1 and trait $j$ in environment $2 ; r_{i 1, i 2}=$ the genetic correlation between trait $i$ in environment 1 and environment $2 ; r_{i, j}=$ the genetic correlation between trait $i$ and trait $j$ within an environment; and $r_{j 1, j 2}=$ the genetic correlation between trait $j$ in environment 1 and environment 2.

The genetic correlation matrix was bent to make it positive definite (Hayes and Hill, 1981). The heritability and genetic standard deviation $\left(\sigma_{A}\right)$ for each trait were based on estimates used in the genetic evaluation for Nordic countries for Holstein and Jersey (Interbull, 2010, 2012a,b, 2014, 2016a,b). These estimates were assumed to be the same for the organic and conventional environments but different for the 2 breeds (Table 3 ). The reason for assuming the same $\sigma_{A}$ for organic and conventional dairy production is that these estimates do not differ very much in the Nordic countries (Sundberg et al., 2009).

Accuracy of direct genomic values (DGV) was calculated using the method in Goddard (2009) as implemented in Buch et al. (2012) for multiple information 
sources. The historical effective population size was assumed to be the same because the linkage disequilibrium did not differ between Holstein and Jersey in a study by J. R. Thomasen (VikingGenetics, Assentoft, Denmark; personal communication). The number of animals in the reference population for both the organic and conventional environments was based on the total number of tested females per breed (J. Kring, VikingGenetics; personal communication). The percentage of organic cows in the reference population was assumed to be the same as the percentage of organic cows in Denmark for
Holstein and Jersey (SEGES, 2018). The DGV accuracy was calculated per trait, breed, and environment (Table 3).

\section{BG}

The following BG were set up: a BG for a conventional environment with Holstein cows (BGConvHol), a BG for an organic environment with Holstein cows (BGOrgHol), a BG for a conventional environment with Jersey cows (BGConvJer), and a BG for an

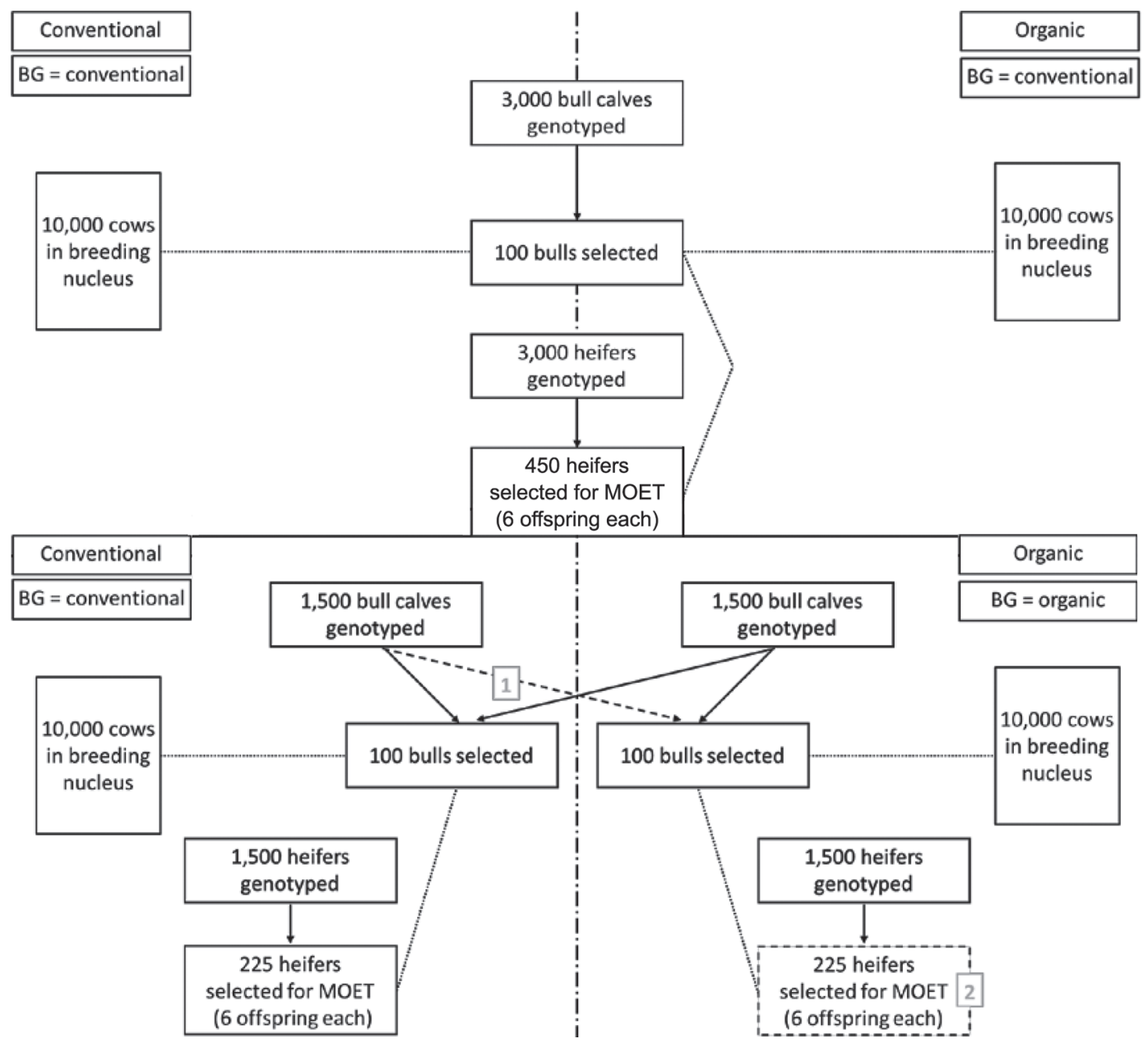

Figure 1. A schematic overview of the breeding program for the simulations with the basic input parameters for Holstein cows. The top picture is the breeding program for scenario Current; all other scenarios are shown in the bottom picture. Arrows indicate that a proportion of animals were selected, and dotted lines indicate that groups of animals were mated. Dashed lines indicate that this part of the breeding program was not applied in all scenarios: selection of bulls to be mated to organic cows from genotyped bull calves from the conventional breeding program was not possible in scenarios Within and Strict (gray box number 1), and multiple ovulation and embryo transfer (MOET) was not possible in scenarios No MOET and Strict (gray box number 2). Scenarios: Current = a conventional breeding goal (BG), MOET, and the possibility to select conventional bulls in the organic breeding program; Organic BG = an organic BG, MOET, and the possibility to select conventional bulls in the organic breeding program; Within = an organic BG, MOET, and no possibility to select conventional bulls in the organic breeding program; No MOET = an organic BG, no MOET, and the possibility to select conventional bulls in the organic breeding program; Strict = an organic BG, no MOET, and no possibility to select conventional bulls in the organic breeding program. 


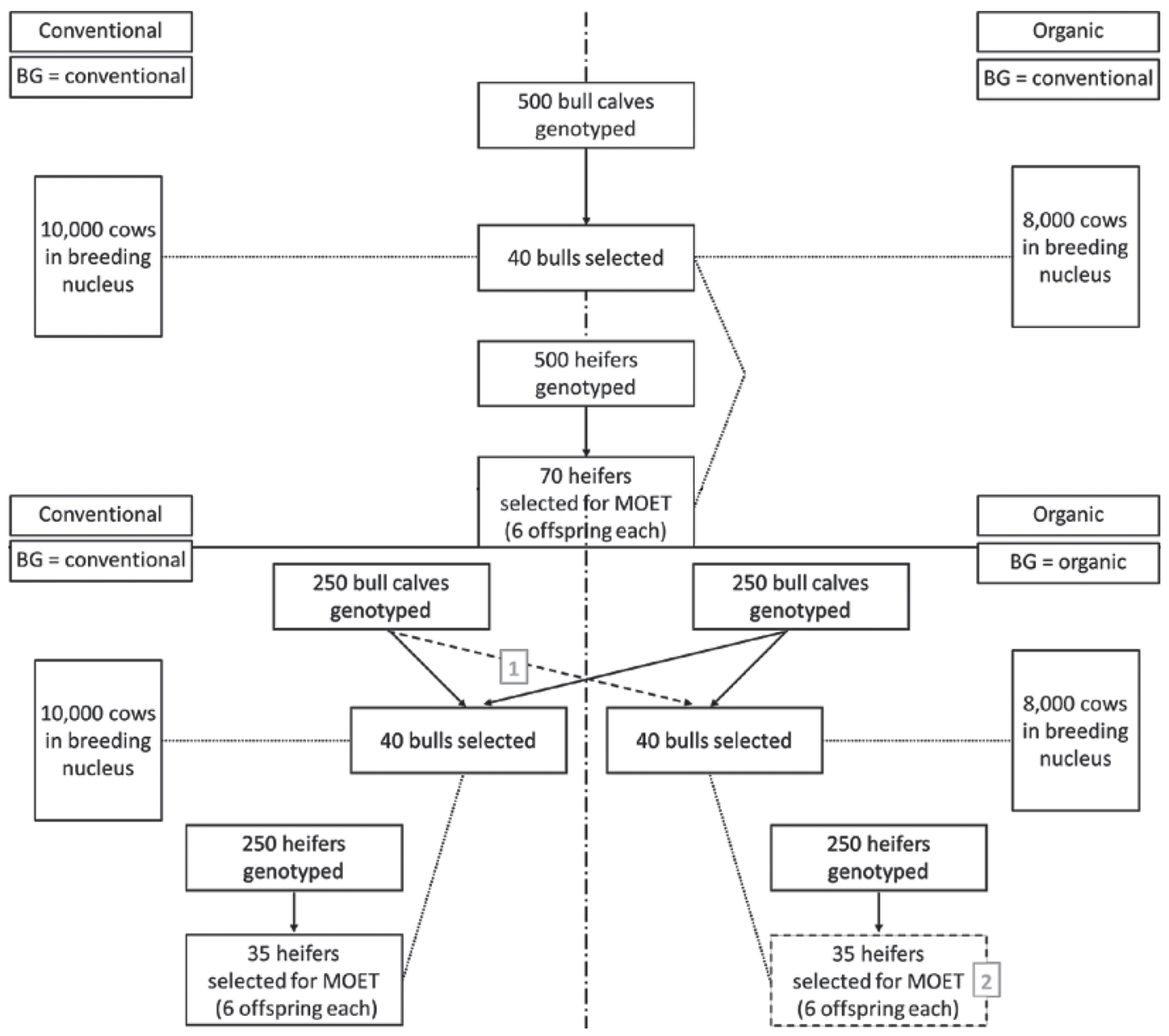

Figure 2. A schematic overview of the breeding program for the simulations with the basic input parameters for Jersey cows. The top picture is the breeding program for scenario Current; all other scenarios are shown in the bottom picture. Arrows indicate that a proportion of animals were selected, and dotted lines indicate that groups of animals were mated. Dashed lines indicate that this part of the breeding program was not applied in all scenarios: selection of bulls to be mated to organic cows from genotyped bull calves from the conventional breeding program was not possible in scenarios Within and Strict (gray box number 1), and multiple ovulation and embryo transfer (MOET) was not possible in scenarios No MOET and Strict (gray box number 2). Scenarios: Current = a conventional breeding goal (BG), MOET, and the possibility to select conventional bulls in the organic breeding program; Organic BG = an organic BG, MOET, and the possibility to select conventional bulls in the organic breeding program; Within = an organic BG, MOET, and no possibility to select conventional bulls in the organic breeding program; No MOET = an organic BG, no MOET, and the possibility to select conventional bulls in the organic breeding program; Strict = an organic BG, no MOET, and no possibility to select conventional bulls in the organic breeding program.

organic environment with Jersey cows (BGOrgJer). These BG were set up based on the economic values calculated for organic and conventional dairy production in Denmark used in the NTM (Sørensen et al., 2018). The economic values per trait unit were multiplied with the $\sigma_{A}$ for each trait. These weights were adjusted to have genetic correlations between each trait and the full BG that were comparable with the correlations between the subindex for fertility, udder health, and the yield index with the NTM (Sørensen et al., 2018; see Table 3 for the BG weights). The genetic correlation between each trait and each BG was calculated using Equation 2 (Buch et al., 2009):

$$
r_{i, j}=\frac{\mathbf{b}_{i}{ }^{\prime} \mathbf{G} \mathbf{b}_{j}}{\sqrt{\mathbf{b}_{i}{ }^{\prime} \mathbf{G} \mathbf{b}_{i} \mathbf{b}_{j}{ }^{\prime} \mathbf{G} \mathbf{b}_{j}}},
$$

where $r_{i, j}=$ the genetic correlation between trait $i$ and BG $j(j=$ BGConvHol, BGOrgHol, BGConvJer, BGOrgJer); $\mathbf{b}_{i}=$ a vector containing 1 for trait $i$ and 0 for the other traits; $\mathbf{b}_{j}=$ a vector containing the $\mathrm{BG}$ weights for $\mathrm{BG} j$; and $\mathbf{G}=$ a matrix containing the genetic correlations between BG traits (Table 2).

This equation was also used to calculate the genetic correlation between the organic and the conventional BG for both Holstein and Jersey cows. In that case, 
Table 2. Correlations between traits used in the simulations (genetic correlations are above the diagonal; residual correlations are below the diagonal)

\begin{tabular}{|c|c|c|c|c|c|c|c|}
\hline \multirow[b]{2}{*}{ Breeding goal } & \multirow[b]{2}{*}{ Trait $^{1}$} & \multicolumn{3}{|c|}{ Conventional } & \multicolumn{3}{|c|}{ Organic } \\
\hline & & $\mathrm{FE}$ & MA & MP & $\mathrm{FE}$ & MA & MP \\
\hline \multirow[t]{3}{*}{ Conventional } & $\mathrm{FE}$ & 1.00 & $0.30^{2}$ & $-0.40^{3}$ & $0.94^{4}$ & $0.29^{5}$ & $-0.38^{5}$ \\
\hline & MA & -0.05 & 1.00 & $-0.35^{3}$ & $0.29^{5}$ & $1.00^{6}$ & $-0.34^{5}$ \\
\hline & MP & -0.10 & 0.05 & 1.00 & $-0.38^{5}$ & $-0.34^{5}$ & $0.97^{6}$ \\
\hline \multirow[t]{3}{*}{ Organic } & $\mathrm{FE}$ & 0.00 & 0.00 & 0.00 & 1.00 & $0.30^{2}$ & $-0.40^{3}$ \\
\hline & MA & 0.00 & 0.00 & 0.00 & -0.05 & 1.00 & $-0.35^{3}$ \\
\hline & MP & 0.00 & 0.00 & 0.00 & -0.10 & 0.05 & 1.00 \\
\hline
\end{tabular}

${ }^{1} \mathrm{FE}=$ fertility; $\mathrm{MA}=$ mastitis; $\mathrm{MP}=$ milk production. Trait parameters for these traits were based on the female fertility index, udder health index, and standardized milk production used in the Nordic Total Merit index.

${ }^{2}$ Buch and Norberg (2008).

${ }^{3}$ Kadarmideen et al. (2000).

${ }^{4}$ Liu et al. (2019).

${ }^{5}$ Calculated with Equation 1.

${ }^{6}$ A. Liu (Aarhus University, Tjele, Denmark; personal communication).

vector $\mathbf{b}_{i}$ contained the $\mathrm{BG}$ weights $\mathrm{BGOrgHol}$ or BGOrgJer and vector $\mathbf{b}_{j}$ contained the BG weights for BGConvHol or BGConvJer. Breeding values were predicted in DMU to take selection decisions based on these BG (Madsen and Jensen, 2013). The method used combined all information in one step.

\section{Statistical Analysis}

For each scenario and breed, genetic gain per year in the organic environment was calculated for each trait in $\sigma_{A}$ units using genetic levels in the last $10 \mathrm{yr}$ of the simulation. Genetic gain per year in the aggregate genotype was calculated in standard deviation units of the BG $\left(\boldsymbol{\sigma}_{\boldsymbol{H}}\right)$ for all scenarios using the BG weights in Table 3. Rate of inbreeding was calculated per generation for each scenario and breed. Genetic gain per year in the aggregate genotype and per trait and rate of inbreeding per generation were compared between scenarios using an ANOVA test. This was followed by Fisher's least significant difference test to make pairwise comparisons using the Agricolae package (de Mendiburu, 2019). All statistical analyses were performed in $\mathrm{R}$ statistical software.

Genetic gain in the aggregate genotype and for each trait was calculated in each alternative scenario relative to genetic gain in scenario Current using Equation 3:

$$
G_{r e l}=\frac{\Delta G_{a l t, i}}{\Delta G_{\text {Current }}} \times 100
$$

where $G_{r e l}=$ relative genetic gain in percentages; $\Delta G_{a l t, i}$ $=$ genetic gain per year in alternative scenario $i(i=$ Organic BG, Within, No MOET, Strict); and $\Delta G_{\text {Current }}$ = genetic gain per year in scenario Current.

Table 3. Input parameters for the traits included in this study

\begin{tabular}{|c|c|c|c|c|c|c|}
\hline \multirow[b]{2}{*}{ Parameter } & \multicolumn{2}{|c|}{ Fertility (d) } & \multicolumn{2}{|c|}{$\begin{array}{c}\text { Mastitis } \\
\text { (percentage units) }\end{array}$} & \multicolumn{2}{|c|}{$\begin{array}{l}\text { Milk production } \\
\text { (kg/305-d lactation) }\end{array}$} \\
\hline & Holstein & Jersey & Holstein & Jersey & Holstein & Jersey \\
\hline Heritability & 0.08 & 0.07 & 0.07 & 0.04 & 0.44 & 0.48 \\
\hline Genetic standard deviation in trait unit & 10.3 & 9.2 & 8.0 & 6.7 & 831.6 & 539.9 \\
\hline BG weight, ${ }^{1}$ conventional ${ }^{2}$ & 105.8 & 42.9 & 71.4 & 53.3 & 158.8 & 103.1 \\
\hline BG weight, ${ }^{1}$ organic ${ }^{3}$ & 86.5 & 32.2 & 88.1 & 75.0 & 118.9 & 78.3 \\
\hline
\end{tabular}

${ }^{1}$ Breeding goal (BG) weights are in $€$ per genetic standard deviation unit.

${ }^{2}$ BGConvHol for Holstein; BGConvJer for Jersey.

${ }^{3}$ BGOrgHol for Holstein; BGOrgJer for Jersey.

${ }^{4} \mathrm{DGV}=$ direct genomic value. Calculated with Goddard (2009) using a genome length $=30$ morgans, effective population size $=750$, number of markers $=38,000$ informative markers, and a percentage of organic cows of approximately $12 \%$ to calculate the number of organic and conventional cows in the reference population. 
Table 4. Correlations between the conventional (Conv) and organic (Org) breeding goal (BG) and between each trait and the whole BG for the simulations with Holstein (Hol) and Jersey (Jer) cattle

\begin{tabular}{|c|c|c|c|c|}
\hline \multirow[b]{2}{*}{ Item } & \multicolumn{2}{|c|}{ Holstein } & \multicolumn{2}{|c|}{ Jersey } \\
\hline & BGConvHol & BGOrgHol & BGConvJer & BGOrgJer \\
\hline Correlation between conventional and organic & \multicolumn{2}{|c|}{0.94} & \multicolumn{2}{|c|}{0.92} \\
\hline Trait & & & & \\
\hline Fertility & 0.41 & 0.48 & 0.18 & 0.26 \\
\hline Mastitis & 0.30 & 0.53 & 0.31 & 0.64 \\
\hline Milk production & 0.58 & 0.39 & 0.70 & 0.43 \\
\hline
\end{tabular}

The percentage of bulls selected for breeding in the organic breeding program but originating from the conventional breeding program was calculated per year for the last $10 \mathrm{yr}$ of the simulation using Equation 4:

$$
P_{\text {conv }}=\frac{N_{\text {conv }}}{N_{\text {conv }}+N_{\text {org }}} \times 100
$$

where $P_{\text {conv }}=$ percentage of selected breeding bulls that originate from the conventional environment; $N_{\text {conv }}=$ number of selected breeding bulls that originate from the conventional environment; and $N_{\text {org }}=$ number of selected breeding bulls that originate from the organic environment.

\section{RESULTS}

\section{Genetic Correlations Between BG}

Genetic correlations between the organic and conventional BG were high for both breeds (Table 4). However, on trait level, clear differences were found between production systems.

\section{Holstein Breeding Program}

Genetic gain per year in the aggregate genotype was significantly different between all scenarios $(P<$ 0.05; Table 5). In scenario Current, genetic gain in $\sigma_{H}$ units was 0.328 . The lowest genetic gain was achieved in scenario Strict, where neither MOET nor the selection of conventional bulls were allowed in the organic environment. Genetic gain in this scenario was $24 \%$ lower compared with scenario Current. When MOET and selection of conventional bulls were both applied (scenario Organic BG), the highest genetic gain in the aggregate genotype was achieved, $1 \%$ higher than in scenario Current. This would thus be the best scenario in terms of genetic gain in the aggregate genotype.

Scenario Current yielded the highest rate of inbreeding per generation, $0.18 \%$ per generation. This estimate was not significantly different from the rate of inbreeding in scenario Within, where the selection of conventional bulls was excluded, and scenario No MOET, where MOET was excluded. The lowest rate of inbreeding per generation was found in scenario Strict, which would thus be the best scenario in terms of rate of inbreeding.

Table 5. Genetic gain and inbreeding in each scenario for the Holstein breed relative to genetic gain and inbreeding in the scenario Current ${ }^{1}$

\begin{tabular}{|c|c|c|c|c|c|}
\hline Scenario $^{2}$ & Breeding goal & MOET & Selection of conventional bulls & Genetic gain ${ }^{3}(\%)$ & Inbreeding $^{4}(\%)$ \\
\hline Organic BG & Organic & Yes & Yes & $101^{\mathrm{a}}$ & $86^{\mathrm{b}}$ \\
\hline Within & Organic & Yes & No & $96^{\mathrm{c}}$ & $97^{\mathrm{a}}$ \\
\hline Strict & Organic & No & No & $76^{\mathrm{e}}$ & $62^{\mathrm{c}}$ \\
\hline
\end{tabular}

${ }^{\mathrm{a}-\mathrm{e}}$ Means within a column with different superscripts differ $(P<0.05)$ according to Fisher's least significant difference test.

${ }^{1}$ Genetic gain in the aggregate genotype in the scenario Current was 0.328 SD units of the breeding goal, and inbreeding was $0.18 \%$ per generation. Both of these estimates were set to $100 \%$.

${ }^{2}$ Current $=$ a conventional breeding goal $(\mathrm{BG})$, multiple ovulation and embryo transfer (MOET), and the possibility to select conventional bulls in the organic breeding program; Organic BG = an organic BG, MOET, and the possibility to select conventional bulls in the organic breeding program; Within = an organic BG, MOET, and no possibility to select conventional bulls in the organic breeding program; No MOET = an organic BG, no MOET, and the possibility to select conventional bulls in the organic breeding program; Strict = an organic BG, no MOET, and no possibility to select conventional bulls in the organic breeding program.

${ }^{3}$ Genetic gain is shown per year for the aggregate genotype in the organic environment only, using the breeding goal weights for the breeding goal in this environment.

${ }^{4}$ Inbreeding was calculated as inbreeding per generation. 


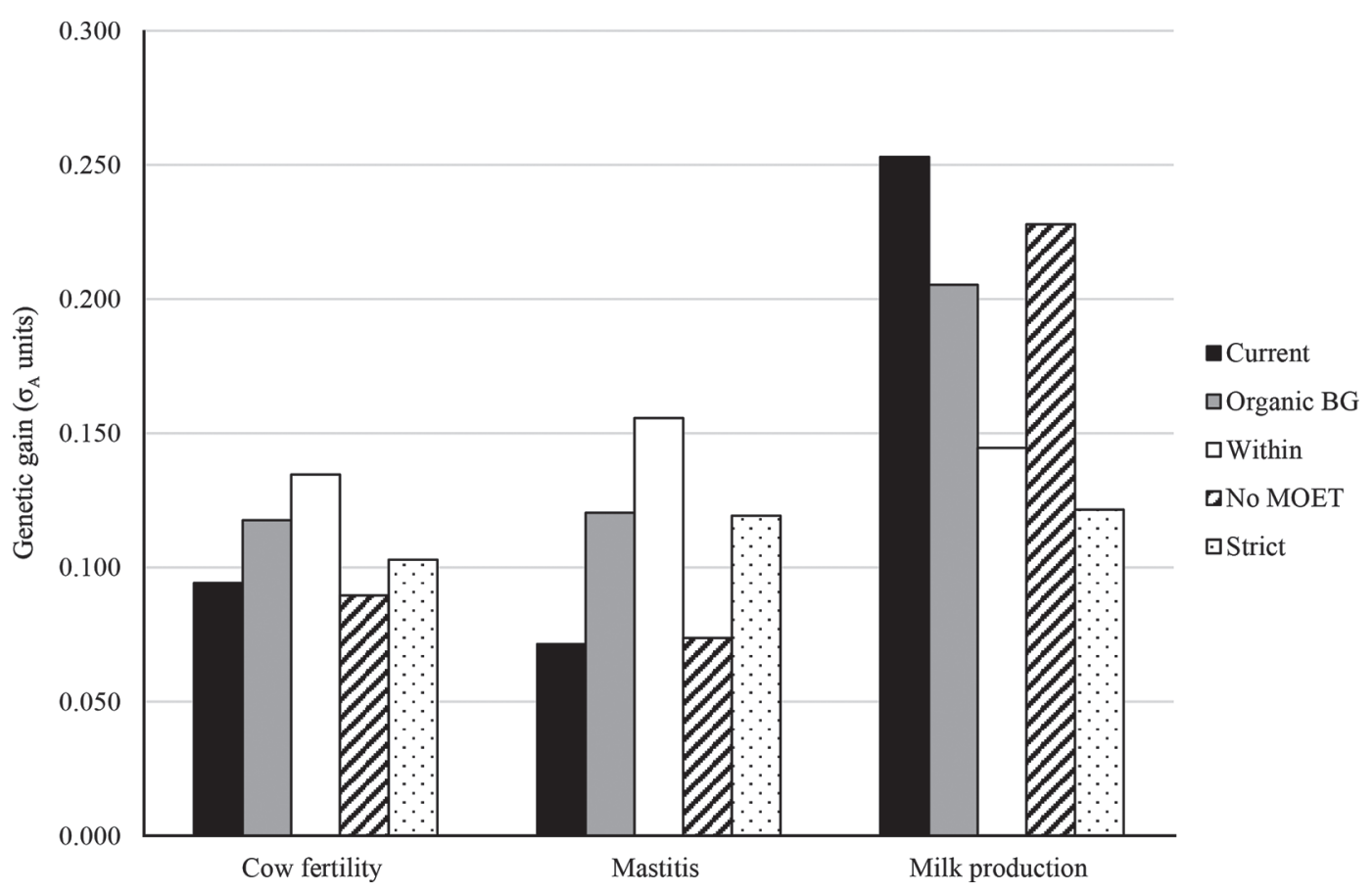

Figure 3. Genetic gain per year in genetic standard deviations units $\left(\sigma_{A}\right)$ for each trait and scenario for the Holstein breed. Current $=$ a conventional breeding goal (BG), multiple ovulation and embryo transfer (MOET), and the possibility to select conventional bulls in the organic breeding program; Organic BG = an organic BG, MOET, and the possibility to select conventional bulls in the organic breeding program; Within = an organic BG, MOET, and no possibility to select conventional bulls in the organic breeding program; No MOET = an organic BG, no MOET, and the possibility to select conventional bulls in the organic breeding program; Strict = an organic BG, no MOET, and no possibility to select conventional bulls in the organic breeding program.

In scenario Within, the highest genetic gain in cow fertility and mastitis was achieved compared with the other breeding strategies (Figure 3). The highest genetic gain in milk production was achieved in scenario Current. Genetic gain in cow fertility and milk production was significantly different in all breeding strategies. Genetic gain in milk production in scenario Strict was merely $48 \%$ of the genetic gain of this trait in scenario Current. Genetic gain in mastitis in scenario Within was $218 \%$ of genetic gain in mastitis in scenario Current. Overall, large differences were found between scenarios for genetic change in the different traits.

\section{Jersey Breeding Program}

There was no significant difference in genetic gain per year in the aggregate genotype between scenarios Current and Organic BG and between scenarios Within and No MOET $(P>0.05$; Table 6$)$. In scenario Current, genetic gain in $\sigma_{H}$ units was 0.270 . The lowest genetic gain in the aggregate genotype was achieved in scenario Strict, where neither MOET nor the selection of conventional bulls were allowed in the organic breeding program. Genetic gain in this scenario was $14 \%$ lower than in scenario Current. Similar to the Holstein breeding program, the highest genetic gain was achieved when MOET and selection of conventional bulls were both applied alongside an environment-specific BG in the organic environment (scenario Organic BG). However, unlike for the Holstein breeding program, this estimate of genetic gain was not significantly different from genetic gain in scenario Current.

Rate of inbreeding per generation was higher in the Jersey breeding program compared with the Holstein breeding program and was highest for scenario Within, $0.5 \%$. However, rate of inbreeding per generation was not significantly higher in this scenario compared with scenario Current. Rate of inbreeding per generation was significantly lower in scenarios No MOET and Strict compared with scenario Current.

As in Holstein, the highest genetic gain in cow fertility and mastitis compared with the other breeding strategies was achieved in scenario Within, and the highest genetic gain in milk production was achieved in scenario Current (Figure 4). Genetic gain in mastitis and milk production was significantly different in all breeding strategies. Large differences were found for genetic change on trait levels. Genetic change in cow 
Table 6. Genetic gain and inbreeding in each scenario for the Jersey breed relative to genetic gain and inbreeding in the scenario Current ${ }^{1}$

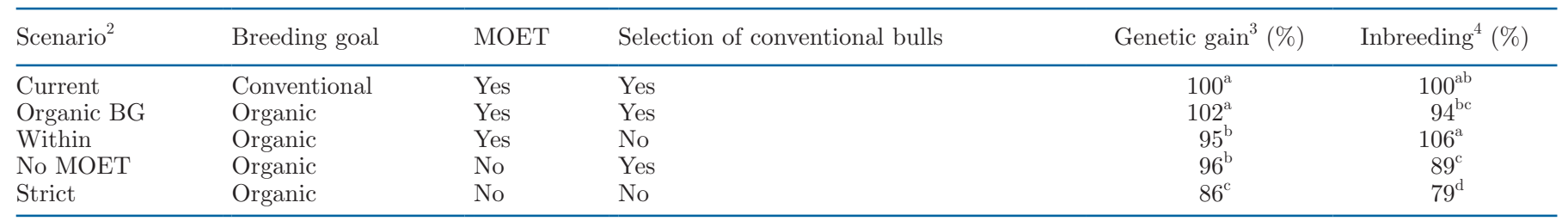

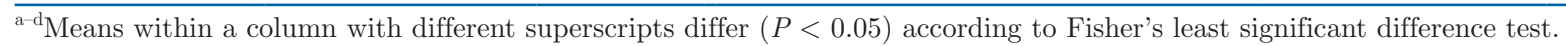

${ }^{1}$ Genetic gain in the aggregate genotype in the scenario Current was $0.270 \mathrm{SD}$ units of the breeding goal, and inbreeding was $0.50 \%$ per generation. Both of these estimates were set to $100 \%$.

${ }^{2}$ Current $=$ a conventional breeding goal (BG), multiple ovulation and embryo transfer (MOET), and the possibility to select conventional bulls in the organic breeding program; Organic BG = an organic BG, MOET, and the possibility to select conventional bulls in the organic breeding program; Within = an organic BG, MOET, and no possibility to select conventional bulls in the organic breeding program; No MOET = an organic BG, no MOET, and the possibility to select conventional bulls in the organic breeding program; Strict = an organic BG, no MOET, and no possibility to select conventional bulls in the organic breeding program.

${ }^{3}$ Genetic gain is shown per year for the aggregate genotype in the organic environment only, using the breeding goal weights for the breeding goal in this environment.

${ }^{4}$ Inbreeding was calculated as inbreeding per generation.

fertility in scenario Within was $-380 \%$ of the genetic change in scenario Current, where genetic change in cow fertility was negative and unfavorable. Genetic gain in mastitis in scenario Within was $560 \%$ of the genetic gain in scenario Current, and genetic gain in milk production was only $59 \%$ of the genetic gain in milk production in scenario Current. Relative differences in genetic gain on trait levels between scenarios were larger for the Jersey breeding program compared with the Holstein breeding program.

\section{Percentage of Breeding Bulls from the Conventional Environment}

In scenarios Within and Strict, selection of bulls for breeding in the organic breeding program and originating from the conventional environment was not possible (Table 7). In scenario Current, the majority of the total number of selected breeding bulls originated from the conventional environment. In scenario Organic BG, this percentage was lowered to 28 to $37 \%$, and in scenario No MOET, large differences between breeds were seen. In the simulations with the Holstein breeding program, $72 \%$ of the bulls selected for breeding in the organic breeding program originated from the conventional environment, whereas this number was $43 \%$ for the Jersey breeding program. Thus, the effect of not using MOET on the percentage of selected conventional bulls was smaller in the Jersey breeding program.

\section{DISCUSSION}

\section{Most Optimal Breeding Strategy}

The most optimal breeding strategy for genetic gain in the aggregate genotype was to implement a specific
BG for organic production and allow for both MOET and the selection of conventional bulls in the organic breeding program. Rate of inbreeding per generation was low in all scenarios and below the recommended $1 \%$ rate of inbreeding (FAO, 2013). Therefore, the rate of inbreeding was not critical when deciding which breeding strategy would be most beneficial. Genetic gain on trait level showed considerable variation between scenarios for both breeds (Figures 3 and 4). Genetic gain in the aggregate genotype was very similar for both breeds between, for example, scenarios Current and Within, where genetic gain was only 4 to $5 \%$ lower in scenario Within. Interestingly, genetic gain on trait level was very different between these scenarios because all bulls that were selected for breeding in the organic breeding program were bred for the organic BG in scenario Within. This BG had more emphasis on functional traits and less on production traits for both the Holstein and the Jersey breeding program, shown by the correlation between each trait and the whole BG (Table 4). The largest difference in genetic gain on trait level in percentages was found for mastitis in the scenarios with the Jersey breeding program, where mastitis in scenario Within was $560 \%$ of the genetic gain in scenario Current. The differences in the scenarios with the Holstein breeding program were less extreme when calculated in percentages, but the differences could still clearly be seen (Figure 3). Even when genetic gain in the aggregate genotype was not significantly different, as for the Current and Organic BG scenarios for the Jersey breeding program, significant and large differences on trait level were found, especially for cow fertility and mastitis. Thus, the most optimal breeding strategy for genetic gain is to implement a specific BG for organic production and to allow for MOET and the selection of conventional bulls. 


\section{Effect of Excluding MOET}

In scenario No MOET, a large proportion of the bulls that were selected for breeding in the organic breeding program originated from the conventional breeding program (Table 7). The reason for this is that conventional bulls had higher breeding values than organic bulls because of the use of MOET in the conventional breeding program. This was especially present in the simulation with the Holstein breeding program, where the size of the MOET was larger than in the simulations with the Jersey breeding program. In these simulations, $72 \%$ of the breeding bulls originated from the conventional environment. Thus, excluding the use of embryo transfer in the organic breeding program resulted in a large percentage of the breeding bulls that were selected for the organic breeding program originating from the conventional environment. The result of selecting mainly conventional bulls was that genetic gain on trait level in the organic breeding program in scenario No MOET resembled genetic gain on trait level in scenario Current, where animals were selected based on the conventional BG. This BG was aimed at improving genetic gain in the conventional breeding program. Hence, the exclu-
Table 7 . The percentage of bulls selected for breeding per year ${ }^{1}$ in the organic breeding program that originated from the conventional environment, per scenario and per breed

\begin{tabular}{llc}
\hline Scenario $^{2}$ & Breed & $\begin{array}{l}\text { Selected bulls originating from } \\
\text { conventional environment (\%) }\end{array}$ \\
\hline Current & Holstein & 54 \\
& Jersey & 53 \\
Organic BG & Holstein & 37 \\
\multirow{2}{*}{ No MOET } & Jersey & 28 \\
& Holstein & 72 \\
& Jersey & 43 \\
\hline
\end{tabular}

${ }^{1}$ The percentage of bulls selected per year was calculated on average for the last $10 \mathrm{yr}$ of the simulation.

${ }^{2}$ Current $=$ a conventional breeding goal $(\mathrm{BG})$, multiple ovulation and embryo transfer (MOET), and the possibility to select conventional bulls in the organic breeding program; Organic BG = an organic BG, MOET, and the possibility to select conventional bulls in the organic breeding program; No MOET = an organic BG, no MOET, and the possibility to select conventional bulls in the organic breeding program. In scenarios Within (an organic BG, MOET, and no possibility to select conventional bulls in the organic breeding program) and Strict (an organic BG, no MOET, and no possibility to select conventional bulls in the organic breeding program), selection of bulls originating from the conventional environment was not possible.

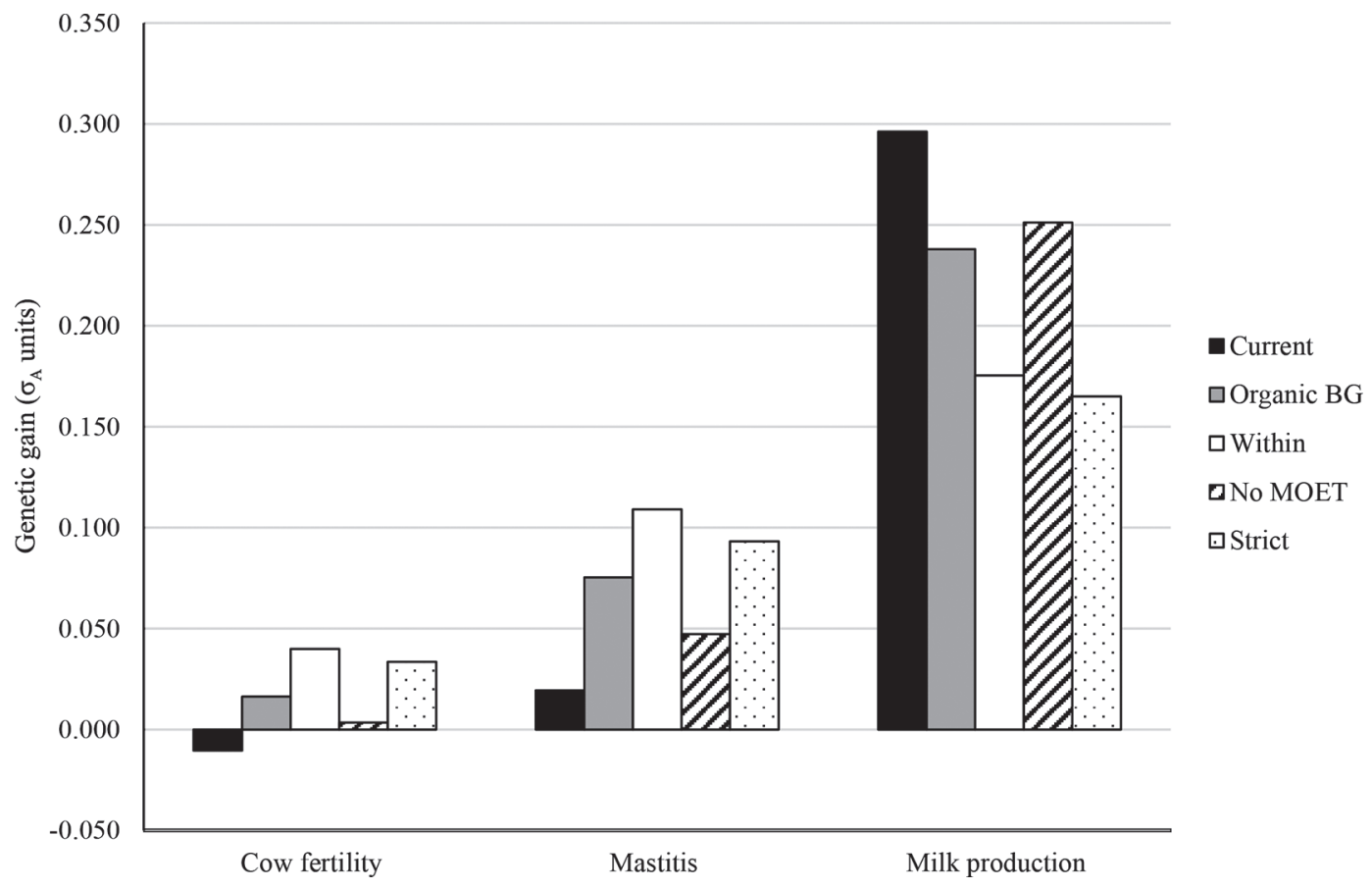

Figure 4. Genetic gain per year in genetic standard deviations units $\left(\sigma_{A}\right)$ for each trait and scenario for the Jersey breed. Current $=$ a conventional breeding goal (BG), multiple ovulation and embryo transfer (MOET), and the possibility to select conventional bulls in the organic breeding program; Organic BG = an organic BG, MOET, and the possibility to select conventional bulls in the organic breeding program; Within = an organic BG, MOET, and no possibility to select conventional bulls in the organic breeding program; No MOET = an organic BG, no MOET, and the possibility to select conventional bulls in the organic breeding program; Strict = an organic BG, no MOET, and no possibility to select conventional bulls in the organic breeding program. 
sion of MOET in the organic breeding program caused individual traits to change in the desirable direction for the conventional BG but not for the organic BG due to the increased selection of conventional bulls. In organic dairy production, improving health is very important when considering the organic principles, an aim that is not reached when using mainly conventional bulls. If this is the consequence of not using MOET, the organic community needs to decide whether this is worth it. Alternatively, a certain loss in genetic gain needs to be accepted if conventional bulls cannot be selected for in the organic breeding program in addition to not using MOET. If the aim is to improve certain traits that are important in organic dairy production, not using embryo transfer might not be the most optimal breeding strategy.

Applying MOET in a breeding scheme generally increases rate of inbreeding due to a higher selection intensity in the female selection path (see, e.g., Meuwissen, 1998; Pedersen et al., 2012). However, when comparing rate of inbreeding in scenario Organic BG and in scenario No MOET in the simulations with the Holstein breeding program, the rate of inbreeding was higher in scenario No MOET (Table 5). This was due to a large group of bulls being selected for breeding that originated from the conventional breeding program (Table 7), which caused fewer bulls to be used in total for breeding in both environments together in this scenario. Thus, the total selected proportion of bulls was smaller, which increased inbreeding. This effect could not be seen in the simulations with the Jersey breeding program because the size of the MOET scheme was much smaller.

\section{Correlation Between BG}

The implementation of an environment-specific BG in the organic breeding program increased genetic gain slightly for both breeds, although this increase was not significant for the Jersey breeding program. The correlation between the organic and the conventional BG was strong for both the Holstein and the Jersey breed and thus expected to be above the previously mentioned break-even correlation. This break-even correlation indicates the specific genetic correlation between BG for different environments above which it is better for genetic gain to have 1 common breeding program with $1 \mathrm{BG}$ for the 2 environments instead of 2 breeding programs with an environment-specific BG for each environment (Mulder et al., 2006; Slagboom et al., 2019). The break-even correlation was estimated to be 0.61 by Mulder et al. (2006) for a breeding program with progeny testing and 0.65 by Slagboom et al. (2019) for a breeding program with genomic selection. Thus, the correlation found between the BG for the 2 environments included in this study is far above the break-even correlation for both breeds. This means that, according to studies concerning the break-even correlation, it would be better for genetic gain to have 1 common breeding program with $1 \mathrm{BG}$ for the organic and the conventional environment. However, the criterion used to estimate the break-even correlation is genetic gain in a breeding objective with the aim to improve genetic gain in 2 environments according to the relative importance of each of these environments. In the current study, the aim was only to improve genetic gain in the organic environment, and thus the criterion used to evaluate which scenario was best did not include genetic gain in the conventional environment. In addition, in scenario Current, the aim was not to increase genetic gain in both environments but just to improve genetic gain in the conventional environment, considering that the BG weights were completely adjusted to the conventional environment. The optimal breeding strategy in the simulations in this study is to implement an environment-specific BG compared with using the same BG as in the conventional environment, even though the correlation between BG for the different environments was very high.

\section{Breeding Program Differences}

The Jersey and the Holstein breeding programs differed for the different input parameters. The Jersey breeding program was smaller in general, meaning the number of bulls and heifers genotyped and selected for MOET was smaller, and the breeding nucleus in the organic breeding program was smaller. Genetic standard deviations of traits were smaller and the accuracy of DGV was lower for the Jersey breed compared with the Holstein breed, especially for the organic breeding program (Table 3). All of these aspects together made it more difficult to achieve genetic change in the simulations with the Jersey breeding program. On the other hand, implementing an organic BG changed genetic change on trait level more in the Jersey breeding program than in the Holstein breeding program. This was due to larger differences between the organic and the conventional BG for the Jersey breed compared with the Holstein breed (Table 4).

The smaller number of heifers selected for MOET in the Jersey breeding program meant that the effect of excluding MOET from the scenarios with the Jersey breeding program had a smaller effect on genetic gain than it had in the scenarios with the Holstein breeding program. In the Jersey breeding program, genetic gain 
in the aggregate genotype was $4 \%$ lower when excluding MOET, whereas this number was $7 \%$ for the Holstein breeding program. For the Holstein breeding program, in scenario No MOET, $72 \%$ of the bulls selected originated from the conventional breeding program, allowing for genetic gain in the aggregate genotype to approach the level of genetic gain in scenario Current. For the Jersey breeding program, the loss in genetic gain due to the exclusion of MOET was already less extreme than for the Holstein breeding program, and thus fewer bulls originating from the conventional breeding program were selected to make up for the loss in genetic gain, about $43 \%$. The result of this can clearly be seen in the genetic gain for individual traits for the Holstein breeding program; genetic gain in individual traits was very similar in scenario No MOET compared with Current. For example, genetic gain in milk production was not significantly different between these 2 scenarios. It is therefore not surprising that excluding both MOET and the selection of conventional bulls in scenario Strict decreased genetic gain in the Holstein breeding program substantially more than in the Jersey breeding program. In this scenario, the loss in genetic gain due to the exclusion of MOET could not be compensated for by the selection of conventional bulls. Thus, the effect of excluding MOET was smaller in the Jersey breeding program.

In the simulations with the Jersey breeding program, more genetic gain in $\sigma_{A}$ units was achieved in milk production compared with the simulations with the Holstein breeding program. This is partly due to a stronger correlation of milk production with the organic BG for the Jersey breed (Table 4). Another reason is that DGV accuracies in the organic environment in the Jersey breeding program were considerably lower compared with the Holstein breeding program, and this was especially true for the 2 functional traits included in this study. The difference in DGV accuracy between the functional traits and milk production caused higher genetic gain in milk production in the organic environment.

\section{Implications}

The results of this study are highly applicable to current-day breeding practices in organic dairy production. Breeding in the desirable direction for organic dairy production without accepting any loss in genetic gain in the aggregate genotype means that scenario Organic BG was the most beneficial scenario to implement. However, the extra genetic gain needs to cover the additional costs to run a separate breeding program, something that is beyond the scope of this study. By not selecting any conventional breeding bulls, the direction of selection will be even more desirable for organic dairy production, but a small loss in genetic gain in the aggregate genotype is also a consequence of this. By collaborating with, for example, organic populations in other countries instead of with the conventional population in the same country, the number of selection candidates could be increased so that genetic gain in the aggregate genotype need not be lower at all compared with the scenario Organic BG. The relative importance of a change in selection intensity and an increase in the accuracy of genomic EBV from collaborating with other organic populations will depend on the actual breeding program and the scope of the collaboration. The use of MOET is debatable due to regulations on MOET in organic production, but the exclusion of the use of MOET causes a large loss in genetic gain in the aggregate genotype. There is no straightforward solution to make up for this loss. The use of sexed semen could be an alternative breeding strategy, but the effect of sexed semen has been shown to be very small compared with the effect of MOET on genetic gain (Pedersen et al., 2012).

\section{CONCLUSIONS}

This study compared different environment-specific breeding strategies for organic dairy production. Implementing a specific BG in the organic breeding program with BG weights that are adjusted to the organic production system increased genetic gain in the aggregate genotype slightly and is therefore a recommendable breeding strategy. Not allowing for MOET and the selection of conventional bulls as breeding bulls in the organic breeding program decreased genetic gain by as much as $24 \%$ and thus is not recommendable, but this is debatable due to the restrictions on the use of MOET in organic dairy production. Assessing genetic gain on trait levels showed that a significant increase in functional traits was possible without a decrease in genetic gain in the aggregate genotype, a finding that is very relevant when breeding for the desired cow in organic dairy production.

\section{ACKNOWLEDGMENTS}

The project SOBcows is part of the Organic RDD 2 program, which is coordinated by the International Centre for Research in Organic Food Systems (ICROFS, Tjele, Denmark). It has received grants from the Green Growth and Development program under the Danish Ministry of Food, Agriculture and Fisheries (Copenhagen). The authors have not stated any conflicts of interest. 


\section{REFERENCES}

Bapst, B., A. Bieber, and E. Haas. 2005. Untersuchungen zur Zuchtstrategie in Schweizer Biol.-Braunviehbetrieben [Analysis of breeding strategies on Swiss organic Barunvieh farms]. https:// www.researchgate.net/publication/238790413_Unter suchungen_ zur_Zuchtstrategie_in_Schweizer_Biol.-Braunviehbetrieben.

Buch, L. H., and E. Norberg. 2008. Genetic analysis of protein yield, udder health, and female fertility in first-parity Danish Holstein cows. Acta Agric. Scand. A Anim. Sci. 58:5-9. https://doi.org/10 .1080/09064700802079136.

Buch, L. H., A. C. Sørensen, J. Lassen, P. Berg, L. G. Christensen, and M. K. Sørensen. 2009. Factors affecting the exchange of genetic material between Nordic and US Holstein populations. J. Dairy Sci. 92:4023-4034. https://doi.org/10.3168/jds.2008-1541.

Buch, L. H., M. K. Sørensen, P. Berg, L. D. Pedersen, and A. C. Sørensen. 2012. Genomic selection strategies in dairy cattle: Strong positive interaction between use of genotypic information and intensive use of young bulls on genetic gain. J. Anim. Breed. Genet. 129:138-151. https://doi.org/10.1111/j.1439-0388.2011.00947.x.

Council of the European Union. 2018. Regulation (EU) 2018/848 of the European Parliament and of the Council of 30 May 2018 on organic production and labelling of organic products and repealing Council Regulation (EC) No 834/2007.

de Mendiburu, F. 2019. Package "Agricolae." Accessed Apr. 26, 2019. https://cran.r-project.org/web/packages/ agricolae/agricolae.pdf.

Dekkers, J. C. M. 2007. Prediction of response to marker assisted and genomic selection using selection index theory. J. Anim. Breed. Genet. 124:331-341. https://doi.org/10.1111/j.1439-0388.2007 .00701.x.

FAO (Food and Agriculture Organization of the United Nations). 2013. In vivo conservation of animal genetic resources. Animal Production and Health Guidelines No. 14. FAO, Rome, Italy.

Goddard, M. 2009. Genomic selection: Prediction of accuracy and maximisation of long term response. Genetica 136:245-257. https: //doi.org/10.1007/s10709-008-9308-0.

Hayes, J. F., and W. G. Hill. 1981. Modification of estimates of parameters in the construction of genetic selection indices ("bending"). Biometrics 37:483-493. https://doi.org/10.2307/2530561.

IFOAM. 2016. Principles of organic agriculture. Accessed Jan. 16, 2017. http://www.ifoam.bio/en/organic-landmarks/principles -organic-agriculture.

IFOAM. 2018. The IFOAM norms for organic production and processing, version 2014. Accessed Jan. 17, 2020. http://www.ifoam.bio/ sites/default/files/ifoam_norms_july_2014_t.pdf.

Interbull. 2010. National Genetic Evaluation Form Holstein Udder Health. Accessed Oct. 4, 2016. https://wiki.interbull.org/ public/Nat_GE_Forms?action $=$ AttachFile $\&$ do $=$ get $\&$ target $=$ DFSHOLuh.rtf.

Interbull. 2012a. National Genetic Evaluation Form Holstein Production Traits. Accessed Oct. 4, 2016. https://wiki.interbull.org/ public/Nat_GE_Forms?action=AttachFile\&do=get\&target= DFSHOLpro.doc.

Interbull. 2012b. National Genetic Evaluation Form Jersey Production Traits. Accessed Sep. 4, 2018. https://wiki.interbull.org/ public/Nat_GE_Forms?action $=$ AttachFile $\&$ do $=$ get \&target $=$ DFSJERpro.doc.

Interbull. 2014. National Genetic Evaluation Form Jersey Udder Health. Accessed Sep. 4, 2018. https://wiki.interbull.org/public/ Nat_GE_Forms?action=AttachFile\&do=get\&target=DFSJERuh .rtf.

Interbull. 2016a. National Genetic Evaluation Form Jersey Fertility Traits. Accessed Sep. 4, 2018. https://wiki.interbull.org/public/ Nat_GE_Forms?action=AttachFile\&do=get\&target=DFSJERff .rtf.

Interbull. 2016b. National Genetic Evaluation Form Holstein Fertility Traits. Accessed Sep. 4, 2018. https://wiki.interbull.org/public/ Nat_GE_Forms?action=AttachFile\&do=get\&target=DFSHOLff .rtf.
Kadarmideen, H. N., R. Thompson, and G. Simm. 2000. Linear and threshold model genetic parameters for disease, fertility and milk production in dairy cattle. Anim. Sci. 71:411-419. https://doi.org/ 10.1017/S1357729800055338.

Kargo, M., J. F. Ettema, L. Hjortø, J. Pedersen, and S. Østergaard. 2015. Derivation of economic values for breeding goal traits in conventional and organic dairy production. Page 403 in Book of Abstracts of the 66th Annual Meeting of the European Association for Animal Production. Wageningen Academic Publishers, Wageningen, the Netherlands.

Kargo, M., L. Hjortø, M. Toivonen, J. A. Eriksson, G. P. Aamand, and J. Pedersen. 2014. Economic basis for the Nordic Total Merit Index. J. Dairy Sci. 97:7879-7888. https://doi.org/10.3168/jds.2013 -7694 .

Krogmeier, D. 2003. Der Ökologische Gesamtzuchtwert für Fleckvieh, Braunvieh und Gelbvieh. [Organic total merit index for Simmental, Brown Swiss and Gelbvieh cattle.] Paper presented at Forschung für den ökologischen Landbau in Bayern,Ökolandbautag der LfL, Triesdorf. Schriftenreihe der Bayerischen Landesanstalt für Landwirtschaft. Bayerische Landesanstalt für Landwirtschaft (LfL), Freising, Germany.

Liu, A., G. Su, J. Höglund, Z. Zhang, J. Thomasen, I. Christiansen, Y. Wang, and M. Kargo. 2019. Genotype by environment interaction for female fertility traits under conventional and organic production systems in Danish Holsteins. J. Dairy Sci. 102:8134-8147. https://doi.org/10.3168/jds.2018-15482.

Madsen, P., and J. Jensen. 2013. A User's Guide to DMU. Version 6 , release 5.2. Aarhus University, Foulum, Denmark.

Meuwissen, T. H. E. 1998. Optimizing pure line breeding strategies utilizing reproductive technologies. J. Dairy Sci. 81:47-54. https:/ /doi.org/10.3168/jds.s0022-0302(98)70153-5.

Mulder, H. A. 2007. Methods to optimize livestock breeding programs with genotype by environment interaction and genetic heterogeneity of environmental variance. PhD Thesis. Wageningen University, Wageningen, the Netherlands.

Mulder, H. A., R. F. Veerkamp, B. J. Ducro, J. A. M. Van Arendonk, and P. Bijma. 2006. Optimization of dairy cattle breeding programs for different environments with genotype by environment interaction. J. Dairy Sci. 89:1740-1752. https://doi.org/10.3168/ jds.S0022-0302(06)72242-1.

Nauta, W. J., R. F. Veerkamp, E. W. Brascamp, and H. Bovenhuis. 2006. Genotype by environment interaction for milk production traits between organic and conventional dairy cattle production in the Netherlands. J. Dairy Sci. 89:2729-2737. https://doi.org/10 .3168/jds.S0022-0302(06)72349-9.

Nicholas, F. W., and C. Smith. 1983. Increased rates of genetic change in dairy cattle by embryo transfer and splitting. Anim. Prod. 36:341-353. https://doi.org/10.1017/S0003356100010382.

Pedersen, L. D., M. Kargo, P. Berg, J. Voergaard, L. H. Buch, and A. C. Sørensen. 2012. Genomic selection strategies in dairy cattle breeding programmes: Sexed semen cannot replace multiple ovulation and embryo transfer as superior reproductive technology. J. Anim. Breed. Genet. 129:152-163. https://doi.org/10.1111/j.1439 $-0388.2011 .00958 . x$.

Pedersen, L. D., A. C. Sørensen, M. Henryon, S. Ansari-Mahyari, and P. Berg. 2009. ADAM: A computer program to simulate selective breeding schemes for animals. Livest. Sci. 121:343-344. https://doi .org/10.1016/j.livsci.2008.06.028.

Rozzi, P., F. Miglior, and K. J. Hand. 2007. A total merit selection index for Ontario organic dairy farmers. J. Dairy Sci. 90:1584-1593. https://doi.org/10.3168/jds.S0022-0302(07)71644-2.

SEGES. 2018. Månedsstatistik 15. August 2018. Accessed Oct. 8, 2018. https://www.landbrugsinfo.dk/Kvaeg/Tal-om-kvaeg/Sider/ mndstatmain.aspx.

Slagboom, M., M. Kargo, D. Edwards, A. C. Sørensen, J. R. Thomasen, and L. Hjortø. 2016. Organic dairy farmers put more emphasis on production traits than conventional farmers. J. Dairy Sci. 99:9845-9856. https://doi.org/10.3168/jds.2016-11346.

Slagboom, M., M. Kargo, A. C. Sørensen, J. R. Thomasen, and H. A. Mulder. 2019. Genomic selection improves the possibility of apply- 
ing multiple breeding programs in different environments. J. Dairy Sci. 102:8197-8209. https://doi.org/10.3168/jds.2018-15939.

Slagboom, M., A. Wallenbeck, L. Hjort $\varnothing$, A. C. Sørensen, L. Rydhmer, J. R. Thomasen, and M. Kargo. 2018. Simulating consequences of choosing a breeding goal for organic dairy production. J. Dairy Sci. 101:11086-11096. https://doi.org/10.3168/jds.2017-14316.

Sørensen, L. P., J. Pedersen, M. Kargo, U. S. Nielsen, F. Fikse, J.-A Eriksson, J. Pösö, R. S. Stephansen, and G. P. Aamand. 2018. Review of Nordic Total Merit Index Full Report November 2018. Accessed Nov. 20, 2018. https://www.nordicebv.info/wp-content/ uploads/2018/11/2018.11.06-NTM-2018-report-Full.pdf.

Sundberg, T., B. Berglund, L. Rydhmer, and E. Strandberg. 2009. Fertility, somatic cell count and milk production in Swedish organic and conventional dairy herds. Livest. Sci. 126:176-182. https://doi .org/10.1016/j.livsci.2009.06.022.

Sundberg, T., L. Rydhmer, W. F. Fikse, B. Berglund, and E. Strandberg. 2010. Genotype by environment interaction of Swedish dairy cows in organic and conventional production systems. Acta Ag- ric. Scand. Sect. A Anim. Sci. 60:65-73. https://doi.org/10.1080/ 09064702.2010.496003.

Thomasen, J. R., A. Willam, C. Egger-Danner, and A. C. Sørensen. 2016. Reproductive technologies combine well with genomic selection in dairy breeding programs. J. Dairy Sci. 99:1331-1340. https: //doi.org/10.3168/jds.2015-9437.

VikingGenetics. 2020. NTM Unlocked - What is NTM? Accessed Jan. 10, 2020. https://www.vikinggenetics.com/about-us/ntm/ntm -unlocked?show $=$ anvp.

\section{ORCIDS}

M. Slagboom (1) https://orcid.org/0000-0002-0296-356X

H. A. Mulder ( https://orcid.org/0000-0003-2124-4787

M. Kargo @i https://orcid.org/0000-0003-1981-2722 